\title{
NORMAL FILTERS GENERATED BY A FAMILY OF SETS
}

\author{
CARLOS AUGUSTO DI PRISCO AND CARLOS ENRIQUE UZCATEGUI
}

(Communicated by Thomas J. Jech)

\begin{abstract}
We study normal filters on the set spaces $\lambda, P_{\kappa}(\lambda),[\lambda]^{\kappa}$, and $(\lambda)^{\kappa}$. We characterize the least normal $\gamma$-complete filter containing a given $\gamma$-complete filter for $\gamma \geq \omega_{1}$. If $₹$ is a $\omega_{1}$-complete filter on any of the set spaces mentioned, the least $\omega_{1}$-complete normal filter containing it is the filter generated by the sets $\left\{x \in E \mid \alpha_{1}, \ldots, \alpha_{n} \in x \rightarrow x \in f\left(\alpha_{1}, \ldots, \alpha_{n}\right)\right\}$ where $f: \lambda<\omega \rightarrow$ 于.
\end{abstract}

1. Introduction. Let $\kappa<\lambda$ be uncountable regular cardinals. The sets $\lambda$, $P_{\kappa}(\lambda)=\{x \subseteq \lambda|| x \mid<\kappa\},[\lambda]^{\kappa}=\{x \subseteq \lambda|| x \mid=\kappa\}$, and $(\lambda)^{\kappa}=\{x \in E \mid \bar{x}=\kappa\}$ are intimately related to certain large cardinal properties. Nevertheless, even without making any large cardinal hypotheses they offer a very rich combinatorial structure interesting by itself.

In this paper we study several normal filters on these spaces built over $\omega_{1}$ complete families of subsets. Throughout the paper we will use $E$ to denote any of the set spaces mentioned above. A family $S \subseteq P(E)$ is $\gamma$-complete if it is closed under intersections of size $<\gamma$. Let $\left\{X_{\xi} \mid \xi<\lambda\right\}$ be a sequence of subsets of $E$. The diagonal intersection of the sequence is defined, as usual, by

$$
\Delta_{\xi<\lambda} X_{\xi}=\left\{x \in E \mid(\forall \xi \in x)\left(x \in X_{\xi}\right)\right\} .
$$

The filter generated by a family $L$ of subsets of $E$ closed under intersections is $[L]=\{A \subseteq E \mid A \supseteq B$ for some $B \in L\}$. The filter $\omega_{1}$-generated by $L$ is the collection of all those subsets of $L$ which contain a countable intersection of elements of $L$.

A filter $\mathcal{F}$ on $E$ is nontrivial if $\phi \notin \mathcal{F}$, and $\mathcal{F}$ is normal if it is closed under diagonal intersections, in other words, if $\left\{X_{\xi} \mid \xi<\lambda\right\} \subseteq \mathcal{F}$ implies $\Delta_{\xi<\lambda} X_{\xi} \in \mathcal{F}$.

A filter is subnormal if it is contained in a nontrivial normal filter.

Given a filter $₹$ on $E$, a subset $S \subseteq E$ is said to have positive $\mathcal{F}$-measure if $S \cap A \neq \varnothing$ for all $A \in \mathcal{F}$. A set $S \subseteq E$ has $\mathcal{F}$-measure 0 if it is not of positive $\mathcal{f}$-measure.

A function $f: E \rightarrow \lambda$ is called regressive on $X \subseteq E$ if for all $x \in X(x \neq \varnothing)$ $f(x) \in x$. A function $f: E \rightarrow A$ taking values in any set $A$ is $\mathcal{F}$-small if for every $a \in A, f^{-1}(a)$ has $\mathcal{F}$-measure 0 .

The following statement is a reformulation of Fodor's theorem $[\mathbf{F}]$ and is due to Jech [Je] for the closed unbounded filter on $P_{\kappa}(\lambda)$ and to Solovay, Reinhardt, and Kanamori [SRK] for the case of ultrafilters on $(\lambda)^{\kappa}$.

Received by the editors September 11, 1985 and, in revised form, June 24, 1986. The contents of this paper were presented at the VII Latin American Logic Symposium held in Campinas, Brazil, in August 1985, sponsored by the Association for Symbolic Logic.

1980 Mathematics Subject Classification (1985 Revision). Primary 03E05, 04A20. 
THEOREM 1.1. Let $\mathcal{F}$ be a filter on $E$. Then $\mathcal{F}$ is normal if and only if for every function $f: E \rightarrow \lambda$ regressive on a positive $\mathcal{F}$-measure set $S \subseteq E$ there is $\alpha<\lambda$ and $S^{\prime} \subseteq S$ also of positive $\mathcal{F}$-measure such that $f(x)=\alpha$ for all $x \in S^{\prime}$.

PROOF. In one direction just follow [Je, 3.2(d)]. The converse follows from the definition of diagonal intersection.

In the next section we prove some facts concerning iterations of the diagonal intersection operation which will be used to characterize the least normal $\omega_{1}$-complete extension of an $\omega_{1}$-complete filter on a set space $E$.

In $\S 3$ we give this characterization and generalize to our spaces a result of Baumgartner, Taylor, and Wagon concerning subnormal filters. The last section contains some examples, including some normal filters properly contained in the closed unbounded filter on $P_{\kappa}(\lambda)$.

2. Iterating diagonal intersections. Let $L$ be any family of subsets of $E$. We will denote by $\Delta L$ the set $\left\{\Delta_{\xi<\lambda} X_{\xi} \mid X_{\xi} \in L\right.$ for all $\left.\xi<\lambda\right\}$. Inductively we define the iterations of the diagonal intersection operation as follows

$$
\Delta^{\alpha+1} L=\Delta\left(\Delta^{\alpha} L\right)
$$

and $\Delta^{\theta} L=\bigcup_{\beta<\theta} \Delta^{\beta} L$, for $\theta$ a limit ordinal (here, $\Delta^{0} L=L$ ). It will be useful to introduce some notation. If $f: \lambda^{n} \rightarrow L$ then

$$
\Delta f=\left\{x \in E \mid \alpha_{1}, \ldots, \alpha_{n} \in x \rightarrow x \in f\left(\alpha_{1}, \ldots, \alpha_{n}\right)\right\} .
$$

Notice that for every $n, \Delta^{n} L$ is just the set $\left\{\Delta f \mid f: \lambda^{n} \rightarrow L\right\}$.

Analogously, if $f: \lambda^{<\omega} \rightarrow L$, define

$$
\Delta f=\left\{x \in E \mid s \in x^{<\omega} \rightarrow x \in f(s)\right\}
$$

and

$$
\Delta^{<\omega} L=\left\{\Delta f \mid f: \lambda^{<\omega} \rightarrow L\right\} .
$$

It is easy to verify that if $L$ is a $\gamma$-complete family of subsets of $E$ then $\Delta L$ is also $\gamma$-complete. The same holds for $\Delta^{<\omega} L$ but not necessarily for $\Delta^{\omega} L$.

THEOREM 2.1. If $L$ is a $\gamma$-complete family of subsets of $E$, and $\gamma \geq \omega_{1}$, then $\left\{\bigcap_{n \in \omega} X_{n} \mid X_{n} \in \Delta^{n} L\right\}$ generates the least $\omega_{1}$-complete filter extending $\Delta^{\omega} L$; moreover, it is $\gamma$-complete.

PROOF. Let $\mathcal{G}$ be the filter generated by the collection $\left\{\bigcap_{n \in \omega} X_{n} \mid X_{n} \in \Delta^{n} L\right\}$. To show that $\mathcal{G}$ is $\gamma$-complete, let $\delta<\gamma$ and let $\left\{Y_{\xi} \mid \xi<\delta\right\}$ be a collection of elements of $\mathcal{G}$. For every $\xi<\delta, Y_{\xi} \supseteq \bigcap_{n \in \omega} X_{n}^{\xi}$ for some sequence $\left\{X_{n}^{\xi} \mid n \in \omega\right\}$ such that $X_{n}^{\xi} \in \Delta^{n} L$ for every $n \in \omega$. Put $Z_{n}=\bigcap_{\xi<\delta} X_{n}^{\xi}$. Since $\Delta^{n} L$ is $\gamma$-complete, $Y_{n} \in \Delta^{n} L$. Clearly,

$$
\bigcap_{\xi<\delta} Y_{\xi} \supseteq \bigcap_{n \in \omega} \bigcap_{\xi<\delta} X_{n}^{\xi}=\bigcap_{n \in \omega} Z_{n} \in \mathcal{G}
$$

If $\mathcal{F}$ is any $\omega_{1}$-complete filter extending $\bigcup_{n \in \omega} \Delta^{n} L$, then $\bigcap_{n \in \omega} X_{n} \in \mathcal{F}$ for each family $\left\{X_{n} \mid n \in \omega\right\}$ such that $X_{n} \in \Delta^{n} L$ for every $n \in \omega$. Therefore the filter generated by $\left\{\bigcap_{n \in \omega} X_{n} \mid X_{n} \in \Delta^{n} L\right\}$ is contained in $\mathcal{F}$. 
3. Normal extensions. A very natural problem is to characterize the least normal filter with a given degree of completeness and containing a given family of subsets of $E$.

Clearly, $\Delta^{\lambda^{+}} L$ generates the least normal filter containing $L$, but our next result shows that if $L$ is an $\omega_{1}$-complete family of subsets of $E$, we only need to look at the first $\omega$ iterations of the diagonal intersection operation to obtain an $\omega_{1}$-complete normal filter extending $L$.

THEOREM 3.1. For every $L \subseteq P(E), \Delta^{<\omega} L$ generates the least normal $\omega_{1}$ complete filter on $E$ extending $L$.

Proof. We show that the family $\Delta^{<\omega} L$ is itself $\omega_{1}$-complete. Let $\left\{X_{i} \mid i \in\right.$ $\omega\} \subseteq \Delta^{<\omega} L$. For each $i \in \omega$ there is a function $f_{i}: \lambda^{<\omega} \rightarrow L$ such that $\Delta f_{i}=X_{i}$. It is easy to code the functions $f_{i}$ into a single function $f$ such that $\Delta f \subseteq \Delta f_{i}$ for each $i$. This can be done, for example, defining

$$
f\left(\alpha_{1}, \ldots, \alpha_{n}\right)=f_{i}\left(\alpha_{1}, \ldots, \alpha_{n_{i}}\right)
$$

where

$$
n=p_{i}^{n_{i}} p_{i+1}^{n_{i+1}} \cdots p_{i+j}^{n_{i+j}} .
$$

To prove that $\Delta^{<\omega} L$ generates a normal filter, let $\left\{f_{\xi} \mid \xi<\lambda\right\}$ be a family of functions, each $f_{\xi}: \lambda^{<\omega} \rightarrow L$. Define $f: \lambda^{<\omega} \rightarrow L$ by

$$
\begin{gathered}
f\left(\alpha_{1}, \ldots, \alpha_{n}\right)=f_{\alpha_{1}}\left(\alpha_{2}, \alpha_{3}, \ldots, \alpha_{n}\right) \text { if } n>1 \\
\text { (and } f(\alpha)=f_{\alpha}(\alpha) \text { otherwise). }
\end{gathered}
$$

Clearly $\Delta f \subseteq \Delta_{\xi}\left(\Delta f_{\xi}\right)$.

Suppose $\overline{\mathcal{F}}$ is a normal $\omega_{1}$-complete filter extending $L$ and strictly contained in $\left[\Delta^{<\omega} L\right]$. Then, there is a function $f: \lambda^{<\omega} \rightarrow L$ such that $\Delta f \in\left[\Delta^{<\omega} L\right]-\mathcal{F}$. The set $E-\Delta f$ is of positive $\mathcal{F}$-measure. Consider the function $h: E-\Delta f \rightarrow \lambda^{<\omega}$ defined by $h(x)=$ some $s \in[x]^{<\omega}$ such that $x \notin f(s)$. By the $\omega_{1}$-completeness of $₹$ there is $n \in \omega$ and $A \subseteq E-\Delta f, A$ of positive $\mathcal{F}$-measure such that $h \uparrow A: A \rightarrow \lambda^{n}$ and for all $x \in A h(x) \in[x]^{n}$. Applying the normality of $\mathcal{F} n$ consecutive times we can find $s \in \lambda^{n}$ and $B \subseteq A, B$ of positive $\mathcal{F}$-measure, such that for all $x \in B$, $x \notin f(s)$ and thus $B \cap f(s)=\varnothing$. This contradicts $f(s) \in L$.

COROLLARY 3.2. If $L$ is a $\gamma$-complete family of subsets of $E$ and $\gamma \geq \omega_{1}$, then $\Delta^{\omega} L \omega_{1}$-generates the least normal $\gamma$-complete filter extending $L$.

ProOF. Let $\mathcal{F}$ be the filter $\omega_{1}$-generated by $\Delta^{\omega} L$. Theorem 2.1 indicates that $\mathcal{F}=\left[\left\{\bigcap X_{n} \mid X_{n} \in \Delta^{n} L\right\}\right]$, and so we only need to show that it is normal. By Theorem 3.1, this will be accomplished if we prove that $\mathcal{F}$ is generated by $\Delta^{<\omega} L$.

Let $A \in \mathcal{F}$; there are functions $f_{n}: \lambda^{n} \rightarrow L, n \in \omega$, such that $A \supseteq \bigcap_{n \in \omega} X_{n}$ where, for each $n \in \omega, X_{n}=\Delta f_{n}$.

Define $f: \lambda^{<\omega} \rightarrow L$ by $f\left(\alpha_{1}, \ldots, \alpha_{n}\right)=f_{n}\left(\alpha_{1}, \ldots, \alpha_{n}\right)$. Then $A \supseteq \Delta f$ and thus $A \in \Delta^{<\omega} L$.

Conversely, if $A \in \Delta^{<\omega} L, A \supseteq \Delta f$ for some $f: \lambda^{<\omega} \rightarrow L$. For each $n \in \omega$, put $f_{n}=f \mid \lambda^{n}$, then $\Delta f=\bigcap_{n \in \omega} \Delta f_{n}$. Since $\Delta f_{n} \in \Delta^{n} L$ for each $n \in \omega$, we conclude that $A \in \mathcal{F}$. 
Baumgartner, Taylor, and Wagon proved in [BTW] a result characterizing, in terms of regressive functions, subnormal, nonprincipal, $\lambda$-complete ideals on a regular cardinal $\lambda$. As a consequence of our previous results this characterization extends to all set spaces $E$.

COROLLARY 3.3. The following are equivalent for an $\omega_{1}$-complete filter $\mathcal{f}$ on $E$ :

(i) $\mp$ is contained in a nontrivial $\omega_{1}$-complete normal filter.

(ii) If $f: E \rightarrow \lambda^{<\omega}$ is regressive (i.e. for all $x \in E, f(x)$ is a finite sequence of elements of $x)$, then it is constant on a set of positive $\mathcal{F}$-measure.

(iii) If $A \in \mathcal{F}$ then there is no $\mathcal{F}$-small regressive function $f: A \rightarrow \lambda^{<\omega}$.

ProOF. For the equivalence of (i) and (ii), let $₹$ be contained in a nontrivial $\omega_{1}$-complete normal filter and let $f: E \rightarrow \lambda^{<\omega}$ be regressive. Suppose $f^{-1}(s)$ is of $\mathcal{F}$-measure 0 for each $s \in \lambda^{<\omega}$. Then $C_{s}=E-f^{-1}(s)$ belongs to $\mathcal{F}$ for each $s \in \lambda^{<\omega}$ and therefore

$$
\Delta_{s} C_{s}=\left\{x \in E \mid s \in x^{<\omega} \rightarrow x \in C_{s}\right\}
$$

is nonempty (since it belongs to the filter $\Delta^{<\omega} \mathcal{F}$ and this is the least $\omega_{1}$-complete normal filter extending $\mathcal{F})$. For $x \in \Delta_{s} C_{s}, f(x) \neq s$ for all $s \in x^{<\omega}$, but this is impossible since $f$ is regressive. Therefore, $f$ must be constant on a set of positive $\mathcal{f}$-measure.

Conversely, if there is no nontrivial $\omega_{1}$-complete normal filter extending $\mathcal{F}$, then $\Delta^{<\omega} \mathcal{F}$ is trivial and thus there is $f: \lambda^{<\omega} \rightarrow \mathcal{F}$ such that $\Delta f=\varnothing$. For every $x \in E$ there is $s \in X^{<\omega}$ such that $x \in f(s)$. Define $h: E \rightarrow \lambda^{<\omega}$ by picking one such $s$ for each $x \in E$. This function is regressive but not constant on any set of positive F-measure.

The equivalence between (ii) and (iii) is equally easy to verify.

In some cases it is possible to generate a normal extension of a family of subsets of $E$ by iterating the diagonal intersection operation a finite number of times.

For example, if $\mathcal{F}$ is a $\lambda$-complete filter on $E$, then $\Delta \mathcal{F}$ is normal. (Given any $f: \lambda^{2} \rightarrow \mathcal{F}$, define $g: \lambda \rightarrow \mathcal{F}$ by $g(\alpha)=\bigcap_{\gamma, \beta \leq \alpha} f(\gamma, \beta)$, then $\left.\Delta g \subseteq \Delta f\right)$.

Also, if we put $C(\phi)=\{x \in E \mid \forall \alpha, \beta \in x \phi(\alpha, \beta) \in x\}$ for any function $\phi: \lambda^{2} \rightarrow \lambda$ we have

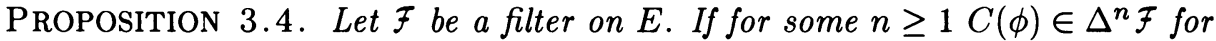
some bijection $\phi: \lambda^{2} \rightarrow \lambda$ then $\Delta^{n} \mathcal{F}$ is normal.

PrOOF. It is enough to show that under the hypothesis of the proposition, for every function $f: \lambda^{n+1} \rightarrow \mathcal{F}$, there is a function $g: \lambda^{n} \rightarrow \mathcal{F}$ such that $\Delta g \cap C(\phi) \subseteq$ $\Delta f$. Let $f: \lambda^{n+1} \rightarrow \mathcal{F}$ and define $g: \lambda^{n} \rightarrow \mathcal{F}$ by

$$
g\left(\alpha_{1}, \ldots, \alpha_{n}\right)=f\left(\phi^{-1}\left(\alpha_{1}\right), \alpha_{2}, \ldots, \alpha_{n}\right) .
$$

Let $x \in \Delta g \cap C(\phi)$. If $\alpha_{0}, \ldots, \alpha_{n} \in x$ then $\phi\left(\alpha_{0}, \alpha_{1}\right) \in x$ and thus,

$$
x \in g\left(\phi\left(\alpha_{0}, \alpha_{1}\right), \alpha_{2}, \ldots, \alpha_{n}\right)=f\left(\alpha_{0}, \ldots, \alpha_{n}\right) .
$$

It can be shown that this is not a necessary condition for the normality of $\Delta^{n} \mathcal{F}$ (see 4.1). 


\section{Some examples.}

4.1. Consider the family of the sets $C_{\alpha}=\{x \in E \mid \exists \beta \in x(\beta>\alpha)\}$. This family generates a $\lambda$-complete filter and therefore, $\left[\Delta\left\{C_{\alpha} \mid \alpha<\lambda\right\}\right]$ is normal.

Note that for the case $E=P_{\kappa}(\lambda)$, the normal filter generated by $\Delta\left\{C_{\alpha} \mid \alpha<\right.$ $\lambda\}$ is properly contained in the closed unbounded filter. This is so because each diagonal intersection of sets of the form $C_{\alpha}$ is closed under arbitrary unions and unbounded. This example confirms the remark after Proposition 3.4, since no diagonal intersection $\Delta_{\xi} C_{\alpha_{\xi}}$ is contained in $C(j)$ for a bijection $j: \lambda \times \lambda \rightarrow \lambda$ (see [C, 2.6]).

4.2. Consider the sets $C_{p}=\{x \in E \mid p \subseteq x\}$, for $p \in P_{\kappa}(\lambda)$. The filter generated by these sets is $\kappa$-complete (but not $\kappa^{+}$-complete). For the case $E=\lambda$ these are just the sets considered in 4.1.

For $E=\mathcal{P}_{\kappa}(\lambda), C_{p}=\hat{p}=\left\{q \in \mathcal{P}_{\kappa}(\lambda) \mid p \subseteq q\right\}$, and by results of $[\mathbf{C}]$,

$$
\begin{aligned}
{\left[\left\{\hat{p} \mid p \in \mathcal{P}_{\kappa}(\lambda)\right\}\right] } & \varsubsetneqq\left[\Delta\left\{\hat{p} \mid p \in \mathcal{P}_{\kappa}(\lambda)\right]\right. \\
& \varsubsetneqq\left[\Delta^{2}\left\{\hat{p} \mid p \in \mathcal{P}_{\kappa}(\lambda)\right]=\right.\text { closed unbounded filter. }
\end{aligned}
$$

The case $E=[\lambda]^{\kappa}$ was studied in [DP.M1] and [DP.M2]. In this case we have $C_{p}=\check{p}=\left\{Q \in[\lambda]^{\kappa} \mid p \subseteq Q\right\}$ and also, by Proposition 3.4, $\Delta^{2}\left\{\check{p} \mid p \in \mathcal{P}_{\kappa}(\lambda)\right\}$ is normal. The situation for $E=(\lambda)^{\kappa}$ is completely different, ZFC $\nvdash$ "there is a nontrivial $\kappa$-complete normal filter on $(\lambda)^{\kappa}$ containing the sets $C_{p}=\left\{Q \in(\lambda)^{\kappa} \mid p \subseteq\right.$ $Q\} . "[D P . M 2]$.

4.3. Consider the family of sets

$$
O(\gamma)=\{x \in E \mid \text { order type of } x>\gamma\}
$$

for $\gamma<\lambda$.

This family is interesting for $E=P_{\kappa}(\lambda)$ and $\gamma<\kappa$. A subset $A \subseteq P_{\kappa}(\lambda)$ is unbounded by final segments if for all $p \in P_{\kappa}(\lambda)$ there is $\alpha<\lambda$ such that $p \cup(\alpha-\bigcup p) \in A$. The filter generated by the closed sets which are unbounded by final segments is properly contained in the closed unbounded filter (see [DP.M2]).

THEOREM 4.3.1. Let $\mathcal{F}_{O}$ be the filter on $P_{\kappa}(\lambda)$ generated by $\{O(\gamma) \mid \gamma<\kappa\}$,

$$
\mathcal{F}_{O} \subsetneq\left[\Delta \mathcal{F}_{O}\right] \subseteq\left[\Delta^{<\omega} \mathcal{F}_{0}\right] \varsubsetneqq \mathcal{F}_{1},
$$

where $\mathcal{F}_{1}$ is the filter generated by the closed sets which are unbounded by final segments.

PROOF. For the first proper inclusion consider the set

$$
A=\left\{p \in \mathcal{P}_{\kappa}(\lambda) \mid \alpha \in p \cap \kappa \rightarrow \alpha+1<\bar{p}\right\} .
$$

Clearly, $A \notin[\{O(\gamma) \mid \gamma<\kappa\}]$, and $A=\Delta_{\xi<\kappa} A_{\xi}$ where $A_{\xi}=O(\xi+1)$ if $\xi<\kappa$ and $A_{\xi}=O(0)$ if $\xi \geq \kappa$.

For the second proper inclusion, consider the set $B=\left\{p \in P_{\kappa}(\lambda) \mid\right.$ for some $\alpha<\lambda, I_{\alpha} \cap p$ contains at least two elements $\}$, where $I_{\alpha}=[\kappa \cdot \alpha, \kappa \cdot(\alpha+1))$. This set is closed and unbounded by final segments, but it does not contain any set in $\Delta^{<\omega}\{O(\gamma) \mid \gamma<\kappa\}$.

We do not know if there is $n \geq 1$ such that

$$
\Delta^{n}\{O(\gamma) \mid \gamma<\kappa\}=\Delta^{\omega}\{O(\gamma) \mid \gamma<\kappa\}
$$


Further study of these and other filters on $P_{\kappa}(\lambda)$ will be undertaken in a forthcoming paper. There it will be shown that, in general, there is no finite bound to the number of iterations of the diagonal intersection operation needed to obtain a normal filter from a filter on $E$.

ACKNOWLEDGMENT. We thank the referee for many suggestions which improved considerably the presentation of our results.

\section{REFERENCES}

[BTW] J. E. Baumgartner, A. D. Taylor and S. Wagon, Structural properties of ideals, Dissertationes Math. 197 (1982).

$[C]$ D. Carr, The minimal normal filter on $P_{\kappa}(\lambda)$, Proc. Amer. Math. Soc. 86 (1982), 316-320.

[DP.M1] C. A. Di Prisco and W. Marek, A filter on $[\lambda]^{k}$, Proc. Amer. Math. Soc. 90 (1984), 591-598.

[DP.M2] _ On the space $(\lambda)^{\kappa}$, Methods in Mathematical Logic (C. A. Di Prisco, ed.), Lecture Notes in Math., vol. 1130, Springer-Verlag, 1985, pp. 151-156.

[F] G. Fodor, Eine Bemerkung zur Theorie de regressiven Funktionen, Acta Sci. Math. (Szeged) 17 (1956), 139-142.

[Je] Thomas Jech, Some combinatorial problems concerning uncountable cardinals, Ann. Math. Logic 5 (1973), 168-198.

[Me] T. K. Menas, On strong compactness and supercompactness, Ann. Math. Logic 7 (1974), 327359.

[SRK] R. Solovay, W. Reinhardt and A. Kanamori, Strong axioms of infinity and elementary embeddings, Ann. Math. Logic 13 (1978), 73-116.

Departamento de Matemáticas, Instituto Venezolano de inVestigaciones Científicas, Apartado 21827, Caracas 1020A, Venezuela 